\title{
Avaliação clínica e laboratorial de meninas com diagnóstico de puberdade precoce central acompanhadas em ambulatório de referência
}

\section{Clinical and laboratorial evaluation of girls with diagnosis of central precocious puberty accompanied in reference ambulatory}

Luana Pontes Vasconcelos Lima ${ }^{1,2}$. Priscila Macêdo Fernandes ${ }^{1,2}$. Luciana Felipe Ferrer Aragão ${ }^{3,1}$. Milena Silva Sousa $^{4,1}$. Renan Magalhães Montenegro Junior ${ }^{5}$. Ana Paula Dias Rangel Montenegro ${ }^{6,1}$. Annelise Barreto de Carvalho ${ }^{7}$

1 Hospital Universitário Walter Cantídio (HUWC), Fortaleza, Ceará, Brasil. 2 Residente de Endocrinologia Pediátrica, Hospital Universitário Walter Cantídio (HUWC), Fortaleza, Ceará, Brasil. 3 Mestrado em Ciências Médicas, médica assistente e preceptora no Ambulatório de Endocrinologia Pediátrica. 4 Mestranda em ciências médicas, médica preceptora no Ambulatório de Endocrinologia Pediátrica. 5 Doutorado em Clínica Médica, Professor Associado, Faculdade de Medicina, Universidade Federal do Ceará (UFC), Gerente de Ensino e Pesquisa dos Hospitais Universitários, Empresa Brasileira de Serviços Hospitalares (EBSERH-UFC), Fortaleza, Ceará, Brasil. 6 Doutorado em Ciências Médicas, médica assistente e preceptora no Ambulatório de Endocrinologia Pediátrica. 7 Doutorado em Saúde da Criança e do Adolescente, médica assistente e preceptora no ambulatório de Endocrinologia Pediátrica.

\section{RESUMO}

Objetivo: descrever o perfil de meninas tratadas com análogo de GnRH quanto aos aspectos clínicos, radiológicos e laboratoriais. Avaliar o efeito do tratamento com análogos de GnRH no índice de massa corpórea (IMC) de meninas com puberdade precoce central. Metodologia: estudo transversal, retrospectivo, de pacientes do sexo feminino, acompanhadas em hospital terciário entre janeiro de 2007 e julho de 2017. Critérios de inclusão: sexo feminino em tratamento com análogo de GnRH. Critérios de exclusão: sexo masculino, LH pós $1^{\text {a }}$ dose $<5$ UI/L. Utilizou-se o coeficiente de correlação linear de Spearman para variáveis contínuas, o teste Qui-quadrado de Pearson para variáveis categóricas, além do Teste t de Student unilateral. O nível de significância estatística adotado para todos os testes foi de 5\% $(\mathrm{p}<0,05)$. Resultados: Foram avaliadas 128 meninas; idade média da telarca: 6,39 $\pm 1,51$ anos; idade média da pubarca: 6,68 $\pm 1,57$ anos; idade média no início do tratamento: 7,95 $\pm 1,22$ anos; média do LH basal: 1,94 \pm

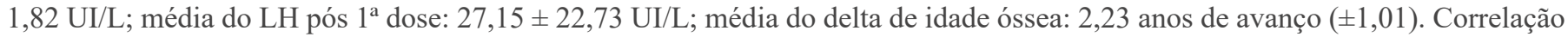
positiva entre o estadiamento puberal e os valores de LH basal e pós estímulo ( $p<0,001)$, LH basal e o delta de idade óssea foi de 0,435 ( $\mathrm{p}<0,001)$, e 11,8\% dos eutróficos evoluíram com sobrepeso e 2,9\% com obesidade, já, das com sobrepeso, 16,1\% tornaram-se obesas. Conclusão: demonstrou-se uma tendência ao aumento do IMC durante o $1^{\circ}$ ano de tratamento tanto no grupo eutrofia quanto sobrepeso. Ressalta-se que a maioria das crianças já iniciou tratamento com sobrepeso ou obesidade (57\%).

Palavras-chave: Puberdade precoce. Índice de massa corporal. Obesidade.

\begin{abstract}
Objective: describe the profile of girls treated with $\mathrm{GnRH}$ analogue for clinical, radiological and laboratory aspects. Also, to evaluate the effect of treatment with GnRH analogues on the body mass index (BMI) of girls with central precocious puberty. Methodology: Retrospective analysis of female patients, followed at a tertiary hospital between January 2007 and July 2017. Inclusion criteria: female patients receiving GnRH analogues. Exclusion criteria: male sex, LH post first dose $<5$ IU/L. Spearman's linear correlation coefficient for continuous variables, the Pearson's Chi-square test for categorical variables, and the unilateral Student's t test were used in the investigation of associations between variables. The level of statistical significance adopted for all tests was $5 \%$ ( $p$ $<0.05$ ). Results: 128 girls were evaluated; mean age of the thelarche: $6.39 \pm 1.51$ years; mean age of the pubarche: $6.68 \pm 1.57$ years; mean age at beginning of treatment: $7.95 \pm 1.22$ years; mean baseline: LH $1.94 \pm 1.82$ IU/L; mean LH post first dose: 27.15 $\pm 22.73 \mathrm{IU} / \mathrm{L}$; median delta bone age: 2.23 years of advancement $( \pm 1.01)$. Positive correlation between pubertal staging and basal and post-stimulus LH values ( $<<0.001$ ), baseline LH and the bone age delta was 0.435 ( $p<0.001)$, and $11.8 \%$ of the eutrophic patients were overweight and $2.9 \%$ were obese, while those with overweight were $16.1 \%$ obese. Conclusion: There was a tendency to increase BMI during the first year of treatment in both the eutrophic and overweight group, but with statistical significance only in the eutrophic group. It is noteworthy that most children have already started treatment with overweight or obesity (57\%).
\end{abstract}

Keywords: Precocious puberty. Body mass index. Obesity.

Autor correspondente: Luana Pontes Vasconcelos Lima, Rua Capitão Francisco Pedro, 1290, Rodolfo Teófilo, Fortaleza, Ceará. CEP: 60430-372. Telefone: +55 85 99961-8474. E-mail: luanapvlima@gmail.com

Conflito de interesses: Não há qualquer conflito de interesses por parte de qualquer um dos autores.

Recebido em: 09 Fev 2018; Revisado em: 17 Mai 2018; Aceito em: 07 Jun 2018. 


\section{INTRODUÇÃO}

A puberdade consiste em um processo fisiológico de transição entre a infância e a fase adulta. ${ }^{1}$ Caracteriza-se por alterações endócrinas e psicológicas que levam à maturação sexual, aceleração do crescimento e ao desenvolvimento da capacidade reprodutiva. ${ }^{1-3}$

Por definição, a puberdade é considerada fisiológica quando se inicia entre 8 e 13 anos nas meninas e entre 9 e 14 anos nos meninos. ${ }^{1-5}$ Entretanto, quando a puberdade se inicia antes dos 8 anos nas meninas ou antes dos 9 anos nos meninos, é considerada precoce. ${ }^{6}$

A puberdade precoce central (PPC) é uma condição rara que comete 1 em cada 5000 a 10000 crianças, sendo mais frequente no sexo feminino com incidência de 20 meninas para cada um menino afetado. Em $90 \%$ dos casos que acometem meninas, a causa é idiopática, enquanto em $50-70 \%$ dos meninos, há comprometimento do sistema nervoso central (SNC). ${ }^{1,5,7}$

Diversas patologias como traumas, infecções, tumores, malformações do SNC, assim como desreguladores endócrinos (substâncias exógenas com ação estrogênica ou androgênica) e mutações genéticas (mutações ativadoras no gene KISS1 ou KISS1R, mutações inativadoras do gene MKRN3) podem levar à puberdade precoce central. ${ }^{2,5}$

O diagnóstico de puberdade precoce central é baseado nos achados clínicos, radiológicos e laboratoriais. São sinais clínicos de desenvolvimento puberal: mamas em estágio M2 de Marshall e Tanner nas meninas e volume testicular $>4 \mathrm{ml}$ ou diâmetro testicular $>2,5 \mathrm{~cm}$ nos meninos, podendo estar associado ao avanço de idade óssea ( $>2$ anos ou $>2,5$ desvios-padrão) e aumento da velocidade de crescimento. ${ }^{6-8}$

A ultrassonografia pélvica deve ser realizada nas meninas com suspeita de PPC, uma vez que é útil tanto no diagnóstico diferencial (cistos, tumores ovarianos) quanto ao avaliar parâmetros como volumes ovarianos e uterino. São considerados puberais volume ovariano maior que $1,8 \mathrm{~cm}^{3} \mathrm{e}$ útero com volume $>4 \mathrm{~cm}^{3}$ ou comprimento uterino $>3,4 \mathrm{~cm} ., 7$

Laboratorialmente, o diagnóstico baseia-se na dosagem do LH basal e após o estímulo com agonista de GnRH ou GnRH exógeno. ${ }^{6,9}$ A dosagem de FSH basal e após estímulo assim como de estradiol não são úteis no diagnóstico de PPC. Valores de $\mathrm{LH}$ basal $>0,3 \mathrm{UI} / \mathrm{L}$ (método quimioluminescência) ou $>0,6$ UI/L (método imunofluorométrico) são considerados puberais e indicam tratamento com análogo de GnRH. Em crianças com valores de LH basal pré-puberais e sinais de PPC, deve ser realizado o teste de estímulo com GnRH exógeno na dose de $100 \mathrm{mcg}$, por via endovenosa, e dosado LH nos tempos 0 , $15,30,40$ e 60 minutos. Alternativamente, pode ser feito a dosagem de LH após estímulo com $1^{\mathrm{a}}$ dose do análogo de GnRH entre 30 e 120 minutos. ${ }^{1,6,7,9}$ Valores de LH pós-teste $>$ $5 \mathrm{UI} / \mathrm{L}$ confirmam o diagnóstico. ${ }^{7}$

O tratamento é feito com análogo de GnRH de ação prolongada e visa interromper a maturação sexual até a idade fisiológica para desenvolvimento puberal, desacelerar a maturação óssea, preservar a altura alvo e evitar problemas psicossociais para criança e familiares. ${ }^{3,4,10-12}$

Assim, este estudo tem como objetivo descrever o perfil das meninas tratadas com análogo de GnRH quanto aos aspectos clínicos, radiológicos e laboratoriais acompanhadas em serviço de referência em endocrinologia pediátrica. Pretende-se ainda avaliar a alteração do z-escore do índice de massa corpórea (IMC) e da relação cintura abdominal pela estatura (RCE) ao longo do tratamento com análogo de GnRH.

\section{MATERIAIS E MÉTODOS}

Estudo transversal, retrospectivo, realizado através de revisão de prontuários dos pacientes com diagnóstico de puberdade precoce central, tratados com análogo de GnRH e acompanhados no ambulatório especializado em Endocrinologia Pediátrica do Hospital Universitário Walter Cantídio (HUWC), Fortaleza, Ceará. A coleta de dados foi realizada entre maio de 2016 e novembro de 2017.

Foram incluídas no estudo meninas com diagnóstico de PPC e tratadas com análogo de GnRH entre janeiro de 2007 e julho de 2017. Critérios de exclusão: sexo masculino, LH após a $1^{\mathrm{a}}$ dose $<5 \mathrm{UI} / \mathrm{L}$, variantes fisiológicas da puberdade, meninas com doenças crônicas que afetam o crescimento (diabetes melitus, hipotireoidismo, doença celíaca, hipocortisolismo) e meninas em uso concomitante de somatropina.

Os dados coletados foram referentes à primeira consulta, início do tratamento, retornos anuais, suspensão do tratamento, menarca e término do crescimento (altura final). Na primeira consulta, registraram-se os dados pessoais (procedência, idade, sexo), antecedentes familiares (estatura dos pais, idade da menarca materna, consanguinidade e história familiar de PPC), dados neonatais (peso ao nascer, comprimento ao nascer e idade gestacional), antecedentes pessoais (adoção, exposição a soja, infecções do SNC, traumatismo cranioencefálico, atraso no desenvolvimento neuropsicomotor, idade da telarca e idade da pubarca).

Foram extraídos os seguintes dados: idade do início do tratamento; valores de LH basal, LH após estímulo e LH controle (coletado no $6^{\circ}$ mês); idade óssea e achados ultrassonográficos (comprimento uterino, volume do útero, volume ovariano) no início; estadiamento puberal, z-escore do IMC, z-escore da estatura, relação cintura abdominal pela estatura no início do tratamento, a cada ano e ao término do bloqueio puberal; velocidade de crescimento anualmente; idade da menarca e estatura final.

Respeitaram-se os preceitos éticos da pesquisa em seres humanos contidos nas diretrizes e normas de pesquisa da resolução 466/2012 do Conselho Nacional de Saúde. Houve preservação da identidade, privacidade e confidencialidade nos dados. A coleta dos dados foi realizada por apenas um pesquisador que teve acesso aos prontuários arquivados. 
Solicitou-se a dispensa do Termo de Consentimento Livre e Esclarecido (TCLE). O estudo obteve aprovação no Comitê de Ética em Pesquisa do HUWC com número de parecer 2.388.070.

A análise estatística das variáveis quantitativas contínuas foi descrita por meio de mediana, percentil 25 e percentil 75 ou média e desvio padrão (DP), conforme normalidade dos dados. Já as variáveis qualitativas categóricas foram descritas através da frequência. Na investigação de associações entre as variáveis, utilizou-se o coeficiente de correlação linear de Spearman para variáveis contínuas, o teste Qui-quadrado de Pearson para variáveis categóricas, além do Teste $\mathrm{t}$ de Student unilateral. O nível de significância estatística adotado para todos os testes foi de $5 \%(\mathrm{p}<0,05)$. As análises estatísticas foram realizadas utilizando o programa estatístico Statistical Package for the Social Sciences (SPSS), versão 22.0 (USA) e software R version 3.3.1.

\section{RESULTADOS}

Foram analisadas 128 meninas com diagnóstico de PPC e tratadas com análogo de GnRH. Dessas, 11 foram excluídas da pesquisa por apresentar LH pós $1^{\mathrm{a}}$ dose $<5 \mathrm{UI} / \mathrm{L}$, porém, essas crianças tinham indicação de tratamento devido à presença de sinais puberais e $\mathrm{LH}$ basal $>0,3 \mathrm{UI} / \mathrm{L}$.

Em relação aos dados pessoais coletados, 52,4\% das meninas eram procedentes da capital; 5,9\% foram adotadas; $1,7 \%$ relataram consanguinidade entre os pais; $21,4 \%$ tinham algum parente de $1^{\circ}$ ou $2^{\circ}$ grau com história de puberdade precoce central; $14,5 \%$ dessas crianças apresentavam atraso no desenvolvimento psicomotor, sendo que em 2 delas foram consequentes a infecções no SNC; e 12,8\% referiram consumo de produtos com soja.

Dos dados neonatais, 9,3\% tinham história de prematuridade e $2,6 \%$ eram pequenos para idade gestacional (PIG) ao analisar peso pela idade gestacional. Aproximadamente 100 crianças não tinham qualquer fator neonatal que pudesse ser correlacionado com a PPC.

A idade de início dos sinais puberais, a idade na $1^{\mathrm{a}}$ consulta com equipe de endocrinologia pediátrica e a idade no início do tratamento estão descritas na Tabela 1 . Vale ressaltar que no início do tratamento apenas $8,6 \%$ das meninas apresentavam estadiamento puberal M2 de acordo com a classificação de Marshall e Tanner, enquanto $71,4 \%$ e $20 \%$ já chegavam em M3 e M4 respectivamente (Tabela 2).

A média do z-escore da estatura no início do tratamento foi de $1,01( \pm 1,16)$ e ao final do tratamento $0,69( \pm 0,97)$. Encontrou-se correlação positiva significativa $(p<0,001)$ entre o z-escore no início e ao término do tratamento. No primeiro ano de tratamento, a média da velocidade de crescimento (VC) foi de $6,2 \mathrm{~cm} / \mathrm{ano}$ $( \pm 2,73)$, enquanto, no $2^{\circ}$ ano, foi de $5,2 \mathrm{~cm} /$ ano $( \pm 2,34)$. A média da relação cintura abdominal pela estatura (RCE) tanto no início quanto no término do tratamento foram de 0,5 . Não houve correlação entre as $\mathrm{VC}$ no $1^{\circ}$ e $2^{\circ}$ ano de tratamento, nem entre a RCE no início e ao final do tratamento com análogo de GnRH. Outros dados antropométricos estão descritos na Tabela 3.
Tabela 1. Dados clínicos de meninas com puberdade precoce central. Serviço de Endocrinologia Pediátrica, Hospital Universitário Walter Cantídio, Universidade Federal do Ceará (UFC), 2007-2017.

\begin{tabular}{lc}
\hline \multicolumn{1}{c}{ Variáveis } & Médias \pm DP (em anos) \\
\hline Idade da telarca & $6,39 \pm 1,51$ \\
Idade da pubarca & $6,68 \pm 1,57$ \\
Idade na $1^{\text {a consulta }}$ & $7,59 \pm 1,47$ \\
Idade no início do tratamento & $7,95 \pm 1,22$ \\
\hline
\end{tabular}

Tabela 2. Estadiamento puberal no início do tratamento em meninas com puberdade precoce central. Serviço de Endocrinologia Pediátrica, Hospital Universitário Walter Cantídio, UFC, 2007-2017.

\begin{tabular}{lc}
\hline $\begin{array}{c}\text { Estadiamento puberal (Marshall } \\
\text { e Tanner) }\end{array}$ & Frequência (\%) \\
\hline M2 & 8,6 \\
M3 & 71,4 \\
M4 & 20 \\
M5 & 0 \\
\hline
\end{tabular}

Tabela 3. z-Escore do IMC ao longo do tratamento em meninas com puberdade precoce central. Serviço de Endocrinologia Pediátrica, Hospital Universitário Walter Cantídio, UFC, 2007-2017.

\begin{tabular}{lcc}
\hline \multicolumn{1}{c}{ Variáveis } & Médias $\pm \mathrm{DP}$ & $P$ \\
\hline Z-Escore do IMC no início & $1,13 \pm 0,91$ & - \\
Z-Escore do IMC no $1^{\circ}$ ano & $1,26 \pm 0,94$ & $p<0,001$ \\
z-Escore do IMC ao final & $1,21 \pm 1,16$ & $p<0,001$ \\
\hline
\end{tabular}

Adotou-se a classificação da Organização Mundial de Saúde (OMS) que divide quanto ao z-escore do IMC em eutrofia (z-IMC $<+1)$, sobrepeso $(+1<$ z-IMC $<+2)$ e obesidade (z-IMC > +2). Das 79 crianças avaliadas em relação ao IMC no início do tratamento, $43 \%$ eram eutróficas, 39,3\% tinham sobrepeso e $17,7 \%$ estavam obesas. Demonstrou-se que, durante o $1^{\circ}$ ano de tratamento $(n=79), 11,8 \%$ dos pacientes eutróficos evoluíram com sobrepeso enquanto 2,9\% com obesidade. Em relação às crianças que iniciaram tratamento com sobrepeso, 16,1\% tornaram-se obesas. Através do teste $\mathrm{t}$ de Student unilateral, descreveu-se correlação positiva significativa $(p<0,001)$ entre a alteração do IMC nos grupos eutrofia para sobrepeso. Foi evidenciado uma tendência de ganho de peso também no grupo sobrepeso, porém, sem significância estatística.

Houve correção positiva estatisticamente significante quando foram correlacionados a variação do z-escore do IMC no início do tratamento com o z-escore do IMC (z-IMC) ao final do $1^{\circ}$ ano e ao término do tratamento, mostrando que realmente há uma tendência de ganho de peso com o uso do análogo de GnRH. 
A média do LH basal foi de 1,94 UI/L $( \pm 1,82)$, enquanto o LH pós $1^{\text {a }}$ dose apresentou média de $27,15 \mathrm{UI} / \mathrm{L}( \pm 22,73)$ pelo método laboratorial quimioluminescência. Evidenciou-se uma forte correção entre os valores de LH basal e LH pós $1^{\mathrm{a}}$ dose $(p<0,001)$. Já a média do LH controle (colhido no $6^{\circ}$ mês) foi de $2,85 \mathrm{UI} / \mathrm{L}( \pm 1,85)$. O coeficiente de correlação de Spearman calculado entre o LH basal e o delta de idade óssea foi de $0,435(p<0,001)$.

Avaliou-se a média do delta de idade óssea (diferença entre a idade óssea no início do tratamento e a idade cronológica), resultando em 2,23 anos de avanço $( \pm 1,01)$. Da avaliação ultrassonográfica, $74,1 \%$ apresentaram volume uterino $>4 \mathrm{~cm}^{3}$, $75,3 \%$ comprimento uterino $>3,4 \mathrm{~cm}$, e $72 \%$ volume ovariano $>1,8 \mathrm{~cm}^{3}$.

O tempo entre o término do tratamento e a menarca foi de, aproximadamente, 12 meses $(n=18)$. De 18 pacientes com estatura final, apenas uma não atingiu a altura alvo, porém, permaneceu dentro do canal familiar. Quando se comparou a previsão de estatura final pelo método de Bailey-Pinneau no início do tratamento, estas pacientes tiveram a altura alvo preservada, mostrando a eficácia do análogo de GnRH em diminuir a velocidade de maturação óssea.

\section{DISCUSSÃO}

A prevalência encontrada no presente estudo foi de 2,6\% de crianças nascidas PIG classificadas pelo peso, o que se aproximou dos achados de Renz et al. nos anos de 2012 e 2013, respectivamente, $3,3 \%$ e 2,5\%. ${ }^{13}$ De acordo com dados da UNICEF publicados em 2013, 11,7\% dos recém-nascidos no Brasil são prematuros. ${ }^{14,15} \mathrm{~A}$ incidência de prematuridade entre as crianças do nosso estudo foi de 9,3\%, mostrando que não houve, na nossa amostra, aumento da incidência por se tratar de crianças com puberdade precoce central.

Foi possível avaliar que a prevalência dos fatores de risco relacionados à puberdade precoce central (recém-nascidos pequenos para idade gestacionais, história familiar de PPC, consanguinidade, história pessoal de retardo no desenvolvimento neuropsicomotor e exposição a soja) condiz com a prevalência já descrita na literatura.

Na nossa amostra, a média de tempo entre os primeiros sinais puberais e a $1^{\text {a }}$ consulta é em torno de 1 ano, sendo que $71,4 \%$ das meninas já chegaram ao ambulatório em estadiamento puberal M3 de Tanner e Marshall, enquanto a média de avanço da idade óssea era de 2,23 anos.

Park et al. descreveram, nos seus achados, uma média de avanço da idade óssea de 1,7 anos. Além disso, foram avaliadas características clínicas e bioquímicas ao longo de 1 ano de tratamento com análogo de GnRH, bem como o impacto do tratamento sobre a obesidade e resistência à insulina em meninas com puberdade precoce. As pacientes apresentaram um aumento no z-escore do IMC e na relação cintura abdominal pela estatura durante o primeiro ano de tratamento, enquanto que não houve alterações no índice HOMA-IR, que avalia resistência insulínica. Quando comparado com o grupo controle, as pacientes com peso normal e excesso de peso exibiram resultados diferentes. Em indivíduos eutróficos, o z-escore do IMC e a RCE aumentaram com o tratamento, mas não apresentaram alterações na resistência insulínica. Já nas crianças com sobrepeso, não houve alterações significativas em relação ao z-escore do IMC e nem à RCE, porém, houve aumento do índice HOMA-IR, o que sugere um aumento na resistência à insulina. ${ }^{16}$

Este achado sugere que o peso do paciente no início do tratamento pode influenciar mudanças na obesidade $\mathrm{e}$ resistência à insulina, demonstrando que o controle de peso de pacientes com PPC variará de acordo com o estado de obesidade do paciente quando o tratamento é iniciado. ${ }^{16}$

Após estudar 383 meninas com PPC, Lee et al. dividiram os indivíduos em três grupos (eutrofia, sobrepeso e obesidade) e avaliou quanto a alteração do IMC ao longo de dois anos de tratamento com análogo de GnRH. A variação do z-escore do IMC foi significativa apenas no grupo das crianças com peso normal no início do tratamento. ${ }^{17,18}$

Em estudo anterior, Brito et al. descreveram uma amostra brasileira com 45 meninas tratadas para $\mathrm{PPC}$, em que não foi evidenciado alteração significativa do IMC ao longo do tratamento, além de mostrar que a maioria das meninas já iniciam tratamento com sobrepeso ou obesidade. ${ }^{4}$ Já Pasquino, em 2008, publicou um estudo em que relatou uma tendência de ganho de peso durante a terapêutica, porém, sem significância estatística. $^{8}$

Chiocca et al. analisaram 17 adolescentes saudáveis tratadas com análogo de $\mathrm{GnRH}$ e que estavam próximas à estatura final (VC $<1 \mathrm{~cm} / \mathrm{ano}$ ), a fim de avaliar quanto ao IMC e a composição corporal. Em relação ao IMC, não houve diferença entre o descrito na época da suspensão do tratamento e no período avaliado. Entretanto, foi descrito aumento da massa gorda corporal, sugerindo que a composição corporal deve ser monitorada até a vida adulta nessas pacientes. ${ }^{19,20}$

Portanto, após a suspensão do tratamento dessas pacientes, torna-se necessário o acompanhamento, a fim de sejam analisados dados em relação à menarca, à estatura final, bem como avaliar em relação ao ganho de peso posterior e às alterações metabólicas decorrentes do excesso de peso.

Na nossa amostra, porém, não foi analisado o aumento da resistência insulínica ao longo do tratamento. Já na RCE, não houve alteração entre o início do tratamento e o término. Vale ressaltar, contudo, que a RCE foi maior do que 0,5 nos dois momentos, mostrando que a maioria das crianças apresenta obesidade abdominal. O aumento no z-escore do IMC, tanto durante o primeiro ano de tratamento quanto no momento da suspensão do tratamento, foi significativo estatisticamente, mostrando que há sim um aumento no ganho de peso ao longo do tratamento. Entretanto, quando as pacientes foram divididas nos grupos eutrofia, sobrepeso e obesidade, verificou-se um aumento significativo do z-escore do IMC apenas naquelas pacientes do grupo eutrofia, o que corrobora com achados já descritos na literatura. 


\section{CONCLUSÃO}

O tratamento com análogo de GnRH se mostrou efetivo em casos de puberdade precoce, uma vez que a estatura alvo das pacientes estudadas foi preservada, reduzindo-se, assim, a progressão acelerada da idade óssea e a rápida velocidade de crescimento.

\section{REFERÊNCIAS}

1. Latronico AC, Brito VN, Carel JC. Causes, diagnosis, and treatment of central precocious puberty. Lancet Diabetes Endocrinol. 2016:4(3):265-74.

2. Abreu AP, Macedo DB, Brito VN, Kaiser UB, Latronico AC. A new pathway in the control of the initiation of puberty: the MKRN3 gene. J Mol Endocrinol. 2015;54(3):131-9.

3. Brito VN, Spinola-Castro AM, Kochi C, Kopacek C, Silva PC, Guerra-Júnior G. Central precocious puberty: revisiting the diagnosis and therapeutic management. Arch Endocrinol Metabolism. 2016;60(2):163-72.

4. Brito VN, Latronico AC, Cukier P, Teles MG, Silveira LF, Arnhold IJ, et al. Factors determining normal adult height in girls with gonadotropin-dependent precocious puberty treated with depot gonadotropin-releasing hormone analogs. J Clin Endocrinol Metab. 2008;93(7):2662-9.

5. Damiani D. Endocrinologia na prática pediátrica. 3ed. Barueri: Manole; 2016.

6. Kletter GB, Klein KO, Wong YY. A pediatrician's guide to central precocious puberty. Clin Pediatr (Phila). 2014;54(5):414-24.

7. Macedo DB, Cukier P, Mendonca BB, Latronico AC, Brito VN. Avanços na etiologia, no diagnóstico e no tratamento da puberdade precoce central. Arq Bras Endocrinol Metab. 2014;58(2):108-17.

8. Pasquino A, Pucarelli I, Accardo F, Demiraj V, Segni M, Nardo D. Long-term observation of 87 girls with idiopathic central precocious puberty treated with gonadotropin-releasing hormone analogs: impact on adult height, body mass index, bone mineral content, and reproductive function. J Clin Endocrinol Metab. 2007;93(1):190-5.

9. Harrington J, Palmert MR, Hamilton J. Use of local data to enhance uptake of published recommendations: an example from the diagnostic evaluation of precocious puberty. Arch Dis Child. 2013;99(1):15-20.

10. Li P, Li Y, Yang CL. Gonadotropin releasing hormone Agonist treatment to increase final stature in children with precocious puberty. Medicine (Baltimore). 2014;93(27):e260.

11. Tam CS, Zegher F, Garnett SP, Baur LA, Cowell CT. Opposing influences of prenatal and postnatal growth on the timing of menarche. J Clin Endocrinol Metab. 2006;91(11):4369-73.
Percebeu-se ainda, uma tendência ao aumento do IMC durante o $1^{\circ}$ ano de tratamento tanto no grupo eutrofia quanto no grupo sobrepeso, devendo-se informar, entretanto, que a significância estatística se deu apenas no grupo eutrofia. Ressalta-se que a maioria das crianças desta amostra (57\%) já iniciou o tratamento com sobrepeso.

12. Zenaty D, Blumberg J, Liyanage N, Jacqz-Aigrain E, Lahlou N, Carel JC. A 6-Month trial of the efficacy and safety of Triptorelin Pamoate $(11.25 \mathrm{mg})$ every 3 months in children with precocious puberty: a retrospective comparison with Triptorelin acetate. Horm Res Paediatr. 2016;86(3):188-95.

13. Renz BM, Cunha KA, Gehm LL, Souza MA, Renner FW. Prevalência de recém-nascidos pequenos para idade gestacional e fatores associados. Bol Cient Pediatr. 2015;04(1):17-21.

14. Cardoso-Demartini A, Bagatin A, Silva R, Boguszewski M. Crescimento de crianças nascidas prematuras. Arq Bras Endocrinol Metabol. 2011;55(8):534-40.

15. Fundo das Nações Unidas para a Infância (UNICEF). Consultoria: pesquisa para estimar a prevalência de nascimentos pré-termo no Brasil e explorar possíveis causas. 2013 [acesso em: 12 dez 2017]. Disponível em: https://www.unicef.org/brazil/pt/br_prematuridade possiveis_causas.pdf

16. Park J, Kim J. Change in body mass index and insulin resistance after 1-year treatment with gonadotropin-releasing hormone agonists in girls with central precocious puberty. Ann Pediatr Endocrinol Metab. 2017;22(1):27-35.

17. Kim S, Kim Y, Lee J, Kim N, Lee W, Ku J, et al. The influence of gonadotropin releasing hormone agonist treatment on the body weight and body mass index in girls with idiopathic precocious puberty and early puberty. Ann Pediatr Endocrinol Metab. 2017;22(2):95-101.

18. Lee HS, Yoon JS, Roh JK, Hwang JS. Changes in body mass index during gonadotropin-releasing hormone agonist treatment for central precocious puberty and early puberty. Endocrine. 2016;54(2):497503.

19. Chiocca E, Dati E, Baroncelli G, Mora S, Parrini D, Erba P, et al. Body mass index and body composition in adolescents treated with gonadotropin-releasing hormone analogue triptorelin depot for central precocious puberty: data at near final height. Neuroendocrinology. 2009;89(4):441-7.

20. Corripio R, Soriano-Guillén L, Herrero FJ, Cañete R, CastroFeijoó L, Escribano A, et al. Changes in body mass index in girls with Idiopathic central precocious puberty under Gonadotropin-Releasing hormone analogue therapy: the Spanish registry. Horm Res Paediatr. 2016;86(3):154-60

\section{Como citar:}

Lima LP, Fernandes PM, Aragão LF, Sousa MS, Motenegro RM Junior, Montenegro AP, et al. Avaliação clínica e laboratorial de meninas com diagnóstico de puberdade precoce central acompanhadas em ambulatório de referência. Rev Med UFC. 2019 jan-mar;59(1):16-20. 